International Journal of Workplace Health Management

\title{
A randomized controlled trial of an app-delivered mindfulness program among university employees: effects on stress and work-related outcomes
}

\author{
Michelle Rich, Jane Ogden and Linda Morison
}

Accepted 7/2/2021 DOI (10.1108/IJWHM-04-2020-0046)

\section{Corresponding author l.morison@surrey.ac.uk}

\footnotetext{
Emerald allows authors to deposit their AAM under the Creative Commons Attribution Noncommercial International Licence 4.0 (CC BY-NC 4.0). To do this, the deposit must clearly state that the AAM is deposited under this licence and that any reuse is allowed in accordance with the terms outlined by the licence. To reuse the AAM for commercial purposes, permission should be sought by contacting permissions@emeraldinsight.com.

For the sake of clarity, commercial usage would be considered as, but not limited to:

o Copying or downloading AAMs for further distribution for a fee;

o Any use of the AAM in conjunction with advertising;

o Any use of the AAM by for promotional purposes by for-profit organisations;

o Any use that would confer monetary reward, commercial gain or commercial exploitation.

Emerald appreciates that some authors may not wish to use the CC BY-NC licence; in this case, you should deposit the AAM and include the copyright line of the published article. Should you have any questions about our licensing policies, please contact permissions@emeraldinsight.com.
} 


\begin{abstract}
Purpose - The purpose of this paper is to evaluate the impact on stress and work-related outcomes of an app-delivered mindfulness-based program (MBP; Headspace $\left.{ }^{\circledR}\right)$ offered to employees in a UK higher education (HE) institution.
\end{abstract}

Design/methodology/approach - Using a randomized waitlist control trial design, participants were randomly allocated to be offered a mindfulness-based program (MBP) immediately for 2 months or after a waiting list period of 2 months (WLC). Data were analyzed using Intention to Treat (ITT ; MBP n=62; WLC n=63); with supplementary analysis restricting to those who completed all measures; $(\mathrm{MBP} n=45 ; \mathrm{WLC} n=56)$ and then further restricting the MBP group to those who completed at least foundation level 1 of the app ; MBP n=31; WLC n=56).

Findings - The ITT analysis showed significant increases in several aspects of mindfulness and a significant reduction in perceived stress but no significant effects for work-related outcomes. Analysis restricted to MBP participants who completed the app foundation course showed a similar pattern but in addition showed significant increases in work-life balance and the emotional aspect of job engagement.

Practical implications - The offer of the Headspace ${ }^{\circledR}$ app in the HE sector may result in reduced perceived stress. If improvements in work-related outcomes are also to be seen, then users need to be encouraged to complete a minimum level of practice.

Originality/value - Effect size estimates for stress and work-related outcomes of an appdelivered MBP contribute to the evolving picture of MBPs in the workplace.

Keywords: Mindfulness; Program; Work; Stress; Work-life balance; Job engagement; University sector 


\section{Introduction}

Over recent years the beneficial effects of Mindfulness Based Programs (MBP) on health and well-being in both clinical (Creswell, 2017) and nonclinical settings (Querstret et al., 2020) have resulted in a burgeoning of such programs being offered in occupational contexts. A strong driver of this is the potential for MBPs to address the increased levels of stress, exhaustion and burnout seen as work has become more complex, more intense and requiring more flexibility and mobility from employees (Vonderlin et al., 2020). Reviews of workplace MBPs have concluded that there is generally consistent evidence for beneficial effects of MBPs on self-reported stress (Allen et al., 2015; Bartlett et al., 2019; Eby et al., 2019; Jamieson and Tuckey, 2017; Lomas et al., 2017; Vonderlin et al., 2020); with growing evidence for beneficial effects on physiological measures (Heckenberg et al., 2018).

Stress reduction, however, is not the only outcome relevant to the workplace setting. For example, a global survey of companies (Buck Consultants, 2016) stated that, in 2016, the top three priorities for wellbeing program objectives in all regions included: improving productivity (59\%), improving employee engagement (56\%), and attracting and retaining employees (54\%). Recent studies of workplace MBPs have broadened their perspective to assess factors such as job performance, job self-efficacy and satisfaction, patient distress, role performance, work engagement, work-life balance and classroom assessments (Eby et al., 2019; Lomas et al., 2017). Good et al., (2015) developed a framework of how MBPs might bring about their effect on these broader outcomes, proposing that the increased attention and decentering brought about by MBPs results in improved cognitive performance, and improved emotion and behavior regulation, which in turn leads to improved work performance, relationships and well-being. However, reviews of the effect of MBPs on such work-related outcomes show that evidence for such effects is relatively sparse and gives a mixed picture (Allen et al., 2015; Bartlett et al., 2019; Lomas et al., 2017; Vonderlin et al., 
2020; ). There is therefore a need to research how the different features of MBPs (platform, content, amount of training) affect different work-related outcomes in different work contexts (Allen et al., 2015; Eby et al., 2019; Jamieson and Tuckey, 2017; Miksch et al., 2015).

The Higher Education (HE) sector is one among many sectors which have seen increases in workplace stressors over the last 20 years. Escalating workloads have led to deterioration in work-life balance, which combined with outcomes-based performance management and increased job insecurity have led to high levels of stress, health problems, job dissatisfaction and high turnover (Kinman and Johnson 2019; Morrish, 2019). Several studies have explored the usefulness of MBPs for university employees (Atkins et al., 2015; Hegney et al., 2020; Kelly, 2017; Klatt et al., 2009; Koncz et al., 2016; Malarkey et al., 2013; Wongtongkam et al., 2017), all using face-to-face interventions; although Hegney et al., (2019) also used video-conferenceing. All reported improvements in stress or other measures of well-being apart from Malarkey et al., (2013). Two studies also included broader work-related outcomes with Koncz et al., (2016) finding significant improvements in the vigor component of job engagement relative to a WLC group and Atkins et al., (2015) finding significant improvements in self-reported job performance relative to two different active control groups.

MBPs were initially delivered face-to-face but this is relatively resource intensive requiring both a teacher and employee time commitment to group meetings which may or may not be held during work hours. Smartphone MBPs provide shorter sessions that can be fitted in according to the user's time availability and therefore allow for more flexibility. There has been a proliferation of smartphone apps designed to deliver mindfulness training (Stratton et al., 2017). Some apps are mindfulness-specific, such as Calm, Wildflowers, VG2 Mindfulness Coach and Headspace ${ }^{\circledR}$ whilst others include elements of mindfulness alongside more generic therapeutic approaches (Clarke and Draper, 2019; Walsh et al., 2019; van 
Emmerik et al., 2018). Such apps promote motivation to practice by giving reminders; providing information on usage and attainment of goals; giving rewards; and sharing experiences within dedicated communities (Fish et al., 2016). More recently, body-sensing devices have been incorporated into mindfulness training giving, for example, neurofeedback or heart rate variability to enhance practice (Balconi et al., 2018; Crivelli et al., 2019).

As a low-cost training program, the commercially available app Headspace ${ }^{\circledR}$ may be well suited to the needs of both HE sector employers and employees. Research to date includes evaluations of the effectiveness of Headspace ${ }^{\circledR}$ for the reduction of mind wandering in the workplace (Bennike et al., 2017), stress reduction and wellbeing (Bostock et al., 2019; Economides et al., 2018), reduction of aggressive behavior (DeSteno et al., 2018), and compassion and burnout (Morrison Wylde et al., 2017). The objective of the present study was to evaluate the effect of offering the app-delivered MBP Headspace ${ }$ to university employees at one UK institution. The app was advertised and offered without any additional input from the researchers or university, instead depending on the motivational aspects of the app. We hypothesized that simply offering the app would be sufficient to bring about changes in stress and possibly broader work-related outcomes. Given the levels of poor work-life balance, job dissatisfaction and high turnover reported among many university staff, we measured work-life balance, job engagement, organizational citizenship behavior and intentions to leave. Acknowledging the fundamental place of research in academia we also measured curiosity.

\section{Method}

\section{Participants}

Participants were recruited from a university in the South of England between August and November 2016. Participants were eligible for inclusion if they were 18 or older; contracted 
to work for at least three days (21.6 hours) per week at the University; had no previous experience of Headspace ${ }^{\circledR}$ and were not under the supervision of a mental health professional for a psychiatric condition. A wide variety of effect sizes was found for stress and workrelated measures in a review of workplace MBPs by Jamieson and Tuckey (2017) so the study was powered to detect a medium effect size $(d=0.50)$ at a significance level of $5 \%$, with $80 \%$ power. Sample size calculations (G*Power 3.1.92) resulted in a target attained sample size of 64 participants per group. Attrition rates vary widely ( $0 \%$ to $56 \%$ ) for MBP studies in the workplace (Michel et al., 2014; Virgili, 2015). The study therefore aimed to recruit 142 participants in each group at baseline (284 in total) allowing for an attrition rate of $50 \%$ postprogram. Of the 278 individuals who accessed the published URL, 107 dropped out at the introductory webpage (before the screening questions). Of the remaining 171 potential participants, 21 did not meet the eligibility criteria (18 due to prior Headspace ${ }^{\circledR}$ use, two due to medical conditions, one due to Headspace ${ }^{\circledR}$ use and medical conditions) and others dropped out at the consent stage or while completing the first questionnaire. Questionnaires were completed at baseline by 125 participants who were then randomized into either the program (MBP; $n=62$ ) or waitlist control (WLC; n=63) groups. Post program, 101 participants completed the same measures with slightly lower retention rates in the MBP group (73\% for the MBP group and $89 \%$ for the WLC group). Figure 1 shows details of participant flow through the study.

[Figure 1]

\section{Procedure}

This study used a randomized waitlist control trial (RCT) to compare two groups: the program group (MBP) was given access to the Headspace ${ }^{\circledR}$ mindfulness-based meditation training app at the start of the study; the wait list control group (WLC) was recruited but not 
given access to the app until after completion of the study. Self-reported measures of mindfulness, stress and work-related outcomes were taken at baseline and two months postprogram. The offer of a free 12-month subscription to Headspace® (value £60) was advertised to university employees via emails, e-board messages, posters and flyers. Qualtrics survey management software was used to administer information sheets, consent forms and questionnaires. After consent was obtained, questions were presented in a fixed order and with forced response. Following the baseline questionnaire, participants were randomly allocated to the MBP or WLC groups using the Randomizer function in Qualtrics and selecting the option which results in approximately equal numbers in each group. Qualtrics was also used to assign Headspace ${ }^{\circledR}$ subscription enrolment codes to participants in the MBP group. After the program, participants were asked to complete the same set of questions again. On completion of the questionnaire, Qualtrics was used to assign Headspace $®$ subscription enrolment codes to participants in the WLC group. Ethical approval for the study was obtained from the university faculty ethical committee (UEC/2016/040/FHMS).

\section{Mindfulness-based program}

Headspace ${ }^{\circledR}$ was the mindfulness-based self-help training application (app) used for this study. Headspace ${ }^{\circledR}$ consists of 30 foundation sessions of 10 minutes each which are available for individuals to use at their convenience. All sessions are repeatable. Headspace ${ }^{\circledR}$ uses audio, video, animations and exercises which incorporate opportunities for breath awareness, body scans, focus, and motivation and intentions. The first foundation level consists of 10 sessions and is free but the remaining two foundation levels and other, optional mindfulness series are available through an annual subscription ( $£ 60$ or $\$ 72$ for a one-year individual subscription). No minimum target number of sessions was set for the two-month program period but as part of the standard Headspace ${ }^{\circledR}$ app, up to four brief emails were sent to inactive participants during the first 15 days after enrolment and three emails sent to 
congratulate participants on achieving milestones within the first ten days. The app was provided without supplementary input from the researchers or university.

\section{Measures}

Participant characteristics (age, sex, education level, marital status), occupation (whether academic and research or back-office and support) and frequency of practicing mindfulness or meditation in the last two years were recorded at baseline.

To provide an accurate record of participant's Headspace ${ }^{\circledR}$ usage, data were collected by Headspace ${ }^{\circledR}$ (date, time and duration of completed sessions and the communication platform used (iOS, Android, Desktop)). If the participant did not click on the subscription link, they did not access the app, so their usage was assigned as zero.

Measures of the following outcome variables were taken at baseline and post-program and for each the reliability was calculated using Cronbach's alpha.

Mindfulness was measured using the Five-Facet Mindfulness Questionnaire - Short Form (FFMQ-SF; Bohlmeijer et al., 2011), which consists of 24-items rated on a 5-point Likert scale (1=never or very rarely true, $5=$ very often or always true; e.g., "I find it difficult to stay focused on what's happening in the present"). The FFMQ-SF measures five aspects of mindfulness and all showed high levels of reliability in this study: observing internal and external stimuli in the present-moment $(\alpha=0.80)$; describing experiences with words $(\alpha=0.82)$; acting with awareness instead of automatically responding while attention is focused elsewhere $(\alpha=0.81)$; nonjudgment of experiences $(\alpha=0.80)$ and nonreactivity of internal experiences $(\alpha=0.79)$. Total mindfulness was also calculated for this study $(\alpha=0.77)$.

Perceived stress was measured using the Perceived Stress Scale 10 (PSS10; Cohen and Williamson, 1988) which consists of 10-items rated on a five-point Likert scale from 0 
(never) to 4 (very often), $(\alpha=0.83)$, (e.g., "In the last month, how often have you felt that you were on top of things?", reverse-scored). The PSS10 stress measure is continuous and the continuous measure was used for the evaluation analysis. However, to give an indication of stress levels in this population at baseline, participants were categorized as having low, moderate or high stress levels using the methodology proposed by Caesar (2016). This compares each participant's perceived stress score with an average for their age group. Low stress is when their score is less than .01 of the age mean; moderate stress is when the difference between their score and the average for their age is between .01 and 11.9; high stress is when the difference is 12 or over.

Work-life balance was measured using 5 items developed by Valcour (2007) rated on a 5-point Likert scale ( $1=$ very dissatisfied, $5=$ very satisfied; $\alpha=0.82)$. The measure incorporates both a cognitive appraisal of balancing work-life demands and an assessment of the emotions or feelings resulting from the appraisal (e.g., "your ability to balance the needs of your job with those of your personal or family life").

Job engagement was assessed using the Job Engagement Scale (JES; Rich et al., 2010), which consists of 18-items measuring three aspects of job engagement: physical $(\alpha=0.80)$, emotional $(\alpha=0.80)$ and cognitive $(\alpha=0.81)$ rated on a 5 -point Likert scale (1=strongly disagree, $5=$ strongly agree). These reflect a physical involvement during the performance of their tasks; an emotional connection to their work or to others in aid of their work; and a cognitive vigilance, focus and attentiveness when performing their work (e.g., "I try my hardest to perform well on my job" (physical); "I feel positive about my job" (emotional); “At work, I pay a lot of attention to my job" (cognitive).

Organizational citizenship behavior (OCB) indicates a willingness to contribute to an organization's success by helping other employees. It was measured using the 8-item 
'behavior toward individuals' subscale of the OCB (Lee and Allen, 2002). Items are rated on a 7-point Likert scale ( $1=$ never, $7=$ always; $\alpha=0.78$; e.g., "Show genuine concern and courtesy toward co-workers, even under the most trying business or personal situations").

Curiosity was measured using the Curiosity and Exploration Inventory-II (CEI-II; Kashdan et al., 2009) which is a 10-item measure with two subscales: 'stretching' $(\alpha=0.80)$, which indicates a motivation to seek out knowledge and experience; and 'embracing' $(\alpha=0.79)$, which indicates embracing novelties, complexities or uncertainties in everyday life. All items are rated on a 5-point Likert scale (1=strongly disagree, 5=strongly agree; e.g., "I frequently seek out opportunities to challenge myself and grow as a person" (stretching); "I enjoy jobs that are excitingly unpredictable" (embracing)).

Intention to quit was measured using a 5-item scale (Wayne et al., 1997), rated on a 7point Likert scale ( $1=$ strongly disagree, $7=$ strongly agree; $\alpha=0.81$; e.g., "As soon as I can find a better job, I'll leave my company").

\section{Data analysis}

Data were downloaded from Qualtrics and analyzed using SPSS version 23 (IBM SPSS, 2014). Preliminary analysis assessed differences in baseline outcome data between the two groups using independent group t-tests. Next, the impact of the program on the outcome measures was compared to the control group using analysis of covariance (ANCOVA) with the baseline measure of the variable as a covariate. This approach is recommended by van Breukelen (2006) as having more power and less bias than a repeated measures approach when randomisation has been successful in producing comparable groups. It also produces an effect size estimate for the comparison of outcomes between the two arms post-intervention which the repeated measures approach does not. Standardized effect sizes for differences between groups were categorized according to Cohen's criteria as $d \geq .2$ indicating small; $d \geq$ 
.5 indicating medium and $\mathrm{d} \geq .8$ indicating large (Cohen, 1977). The impact of the program was assessed in three ways. The main analysis used an Intention to Treat (ITT) approach imputing baseline values for post-program values where post-program values were missing $(27 \%(n=17)$ of the MBP group and $11 \%(n=8)$ of the WLC). Two supplementary analyses were conducted. First only those who had no missing data post-program (completers) were included; and second only those in the MBP group who had completed at least the first foundation course of Headspace® (practitioners) were included. Analysis of participants' profile, occupational characteristics and frequency of mindfulness or meditation characteristics showed no significant differences between completers and non-completers groups, or practitioners and non-practitioners groups (further details available from authors).

\section{Results}

Participant characteristics by group are shown in Table 1. Overall, 69.6\% of participants were female, $78.4 \%$ were graduates and $11.3 \%$ had previous experience with mindfulness or meditation. Over a third of participants (36\%) were categorized as having high stress levels with $51.2 \%$ having moderate stress levels. There were no significant differences between the MBP and WLC groups in any participant characteristics (Table 1) or baseline outcome measures (Table 2) indicating that the two groups were comparable prior to the delivery of the MBP.

[Tables 1 and 2]

Headspace ${ }^{\circledR}$ usage varied greatly within the MBP group (Table 3) with 17 participants (27\%) not accessing the link to the Headspace ${ }^{\circledR}$ app. The maximum duration of practice was 14.6 hours with the median duration being 1.7 hours. Among those who completed both the baseline and post-intervention measures (completers) the median was 3.0 hours and among those who completed at least the first foundation course (practitioners) the median was 5.2 
hours. The vast majority of Headspace ${ }^{\circledR}$ usage was for the first three foundation courses but in addition 21 different Headspace® topics were accessed. Each was accessed by between 13 participants apart from "Focus", "Sleep" and "SOS" which were accessed by 5, 7 and 4 participants respectively.

[Table 3]

The main analysis examined the effect of the intervention using an ITT approach. Table 4 shows unstandardized effect sizes with 95\% confidence intervals, p-values and standardized effect estimates (Cohen's d) for the comparison between the MBP and WLC groups. These indicate significant improvements in aspects of mindfulness relating to acting with awareness $(\mathrm{d}=.51$, medium effect size $)$, nonreactivity $(\mathrm{d}=.39$, small effect size $)$ and the total mindfulness score $(\mathrm{d}=.50$, medium effect size $)$ for those offered Headspace ${ }^{\circledR}$. No significant differences were found for the describing, observing and nonjudgement aspects of mindfulness. Participants offered the MBP showed a significant reduction in perceived stress $(\mathrm{d}=-.39$, small effect size). No significant differences were found for any of the work-related measures.

[Table 4]

Supplementary analyses were conducted including only those who completed measures at both baseline and post-intervention ("completers"). Table 5 shows that among completers, comparisons between the MBP and WLC groups gave a similar pattern of results to the ITT analysis. There were significant differences between groups in mindfulness scores relating to acting with awareness $(\mathrm{d}=.58)$, nonreactivity $(\mathrm{d}=.51)$ and the total mindfulness score $(\mathrm{d}=$ .67). All these effect sizes were medium and larger than for the ITT analysis. In addition, those offered the MBP also reported higher scores for the mindfulness aspect, nonjudgment $(\mathrm{d}=.47$, small effect size). A significant difference was again found for perceived stress with 
a larger effect size than in the ITT analysis $(\mathrm{d}=-.52$, medium effect size $)$. Again no significant differences were found for any of the work-related measures.

\section{[Table 5]}

A final set of analyses was conducted comparing MBP group participants who had completed at least the first foundation course of Headspace ${ }^{\circledR}$ ("practitioners") with the WLC group (Table 5). The practitioners showed significant improvements in mindfulness scores relating to acting with awareness $(\mathrm{d}=.57)$, nonreactivity $(\mathrm{d}=.50)$, nonjudgment $(\mathrm{d}=.70)$ and the total mindfulness score $(\mathrm{d}=.77)$ compared to the WLC group. This was similar to the previous analyses and again effect sizes were medium. As in the previous analyses, a significant reduction in perceived stress, with a medium effect size $(d=-.55)$, was found in the MBP group relative to the WLC group. In addition, significant differences between the groups were found for two of the work-related measures. Work-life balance $(\mathrm{d}=.46)$ and the emotional aspect of job engagement $(d=.46)$ were both significantly higher in the practitioner group compared to the WLC group.

\section{Discussion}

The present study aimed to evaluate the effectiveness of an app-delivered MBP (Headspace $\left.{ }^{\circledR}\right)$ on stress and work-related outcomes in university employees in the HE sector. In this setting the offer of the app was associated with an increase in mindfulness; primarily acting with awareness, nonreactivity and, to a lesser extent, nonjudgement. Little or no effect was found for the mindfulness facets observing and describing. The hypothesis that simply offering the app would result in improvement in self-perceived stress was supported. For the broader work-related outcomes there was evidence of an effect on work-life balance and the emotional component of job engagement but only in the analyses restricted to those who had completed the foundation level of Headspace ${ }$. No significant effect of the Headspace $₫$ app 
was found for the other aspects of job engagement, or for citizenship, curiosity or intention to quit.

The app was offered in this study without additional input from the researchers or employers, relying on the in-built motivators of the app to encourage practice. Not unexpectedly this led to high variability in the amount of usage ranging from not accessing the link up at all up to 14.6 hours of practice. The median practice hours appear low compared with what might be expected during a 6 to 8 week face-to-face program and the variability means that the potential benefits are likely to vary substantially between participants. However, despite this, intervention effects were apparent.

The increase in mindfulness found in this study is consistent with much research using MBPs and indicates that an app, specifically Headspace® in this case, can be an effective delivery method of mindfulness training (Economides et al., 2018; Huberty et al., 2019; Wasil et al., 2019). Interestingly, mindfulness components showing significant improvements in past research vary and this may reflect differences in emphasis within different MBPs. The two components showing the greatest change in the current study (acting with awareness and nonreactivity) were the same as those found by Morrison Wylde et al. (2017) who similarly used Headspace ${ }^{\circledR}$. They suggested that this might be due to Headspace® placing greater focus on scanning the body and environment without judgment than traditionally delivered mindfulness interventions.

Reviews of previous research have generally found evidence for the beneficial effects of a variety of MBPs on stress (Allen et al., 2015; Bartlett et al., 2019; Eby et al., 2019; Jamieson and Tuckey, 2017; Lomas et al., 2017; Vonderlin et al., 2020). The improvements in perceived stress found in this study show that simply offering a commercial smartphone app without additional employer input can result in beneficial changes. Given the high stress 
levels found within the HE sector (Kinman and Johnson 2019; Morrish, 2019) and the effect size of the reduction found in this this study, offering Headspace ${ }^{\circledR}$ is a promising approach to reducing these high levels. However, as Jamieson and Tuckey caution, interventions such as these should not be used to place responsibility for addressing stress solely on individuals, without also implementing strategies at the organizational level to address the underlying causes of stress. LaMontagne et al., (2014) similarly argue that there is a persisting overemphasis on individual directed intervention. They call for an integrated approach to workplace mental health whereby employers need to reduce work-related risk factors and develop positive aspects of work as well as implementing interventions to directly address mental health issues.

There is relatively little research on the broader, work-related outcomes of MBPs (Vonderlin et al., 2020) and this study contributes effect size estimates for several different outcomes for an app-delivered MBP. Significant effects on work-life balance and the emotional aspect of job engagement were found only when restricting the intervention group to those who had a minimal level of practice. This suggests that input may be needed from employers to encourage practice if improvements in these broader outcomes are to be seen. The Headspace® Foundation Level 1 is a relatively low dosage (ten meditations of 10 minutes over two months) but the median hours practiced in this group was 5.2 indicating that many had completed substantially more practice than the Foundation level. Improvements in work-life balance and job engagement following workplace MBPs have been found previously. A study of work-life balance by Michel et al., (2014) which used a three-week on-line MBP with participants from a variety of occupations also found a significant effect. Studies of work engagement have used a variety of measures and most found increases following an MBP (Atkins et al., 2015; Gunasekara and Zheng, 2018; Koncz et al., 2016; Leroy et al., 2013; West et al., 2014) although one study did not find an effect on 
work engagement but also did not find any changes in levels of mindfulness (van Berkel et al., 2014).

There was no clear evidence of improvement in the other work-related measures, although effect sizes for some (e.g. intention to quit $\mathrm{d}=-.40 ; \mathrm{p}=.078$ and the embracing aspect of curiosity $\mathrm{d}=.35 ; \mathrm{p}=.110$ ) suggest that differences for these variables might become apparent in studies with greater sample sizes. It is interesting that no improvements were seen in citizenship behaviours following the MBP. It is possible that implementing strategies to improve work-life balance may reduce the willingness to help work colleagues. Further, mindfulness could increase awareness of job stresses or other negative aspects of the job for some people which may result in negative effects on some of the other work-related measures.

\section{Limitations and Future Research}

There are some limitations of this study, however, that need to be addressed. While power was adequate to detect a medium effect size for the ITT analyses, the power was lower for the completers and practitioners analyses. The measures used in this study have sound psychometric properties but are all self-reported and therefore susceptible to the biases inherent in such outcomes (Podsakoff et al., 2003). In addition, all participants were aware that the study was evaluating a commercially available MBP which they were accessing for free and this may have resulted in socially desirable responses. The study utilized a wait list control rather than an active control whereby participants engage in an alternative program and this limits the conclusions that can be drawn. The sample was drawn from a single university in the South of England and whilst participants represented a variety of job roles within the academic, back-office and support functions of the university, the experiences of employees at this one university may not reflect those across the wider HE sector. Finally, 
although the wait list control participants were asked not to use the app during the control period, we have no evidence whether this was adhered to.

Further research could address the limitation of self-reported outcomes, for example by assessing stress physiologically through body-worn technology and by more objective work related measures such as productivity, days off sick and actual turnover. Future research could also explore variability in response to the Headspace ${ }^{\circledR}$ app to identify which participants benefit most from its use in terms of both app characteristics (e.g., Topic focus, duration of sessions etc.), personal demographics (e.g., age, gender, role etc.) and which components of the app (e.g., Body scan, breath analysis, audio, video etc.) relate to which changes in outcomes. This would enable more tailored use of the app to maximize its effectiveness. This study provides some information on which aspects of mindfulness are increased by a particular MBP, alongside effects on stress and broader work-related outcomes. However, more research is needed on the mechanisms by which different types of MBP affect the facets of mindfulness and how these in turn affect stress and different work outcomes.

In conclusion, this study shows that offering a relatively cheap commercially available app such as Headspace ${ }^{\circledR}$ without further input from the employer can increase mindfulness and reduce perceived stress among university employees. However, to attain improvements in broader work-related outcomes input may be needed from employers to encourage a minimum level of practice. Among those who practiced for a minimum of ten $\mathrm{x}$ 10 minute sessions improvements in work-life balance and the emotional aspect of job engagement were found. 


\section{Acknowledgements}

The authors would like to thank participants for their time. We would also like to thank Headspace ${ }^{\circledR}$ who provided the facility for the university to give one free subscription to study participants. Headspace ${ }^{\circledR}$ did not fund any members of the research team, nor were they involved in the design of the study, data collection, data analysis or write up of the paper. They did provide the meta data from the app usage (date, time and duration of completed sessions) and the communication platform used (iOS, Android, Desktop). 


\section{References}

Allen, T. D., Eby, L. T., Conley, K. M., Williamson, R. L., Mancini, V. S., and Mitchell, M. E. (2015) What do we really know about the effects of mindfulness-based training in the workplace? Industrial and Organizational Psychology, Vol. 8 No. 4, pp. 652-661. https://doi.org/10.1017/iop.2015.95

Atkins, P. W. B., Hassed, C., and Fogliati, V. J. (2015) Mindfulness improves work engagement, wellbeing and performance in a university setting. In R. J. Burke, K. M. Page, and C. L. Cooper (Eds.), Flourishing in life, work and careers: Individual wellbeing and career experiences. (pp. 193-210). Cheltenham, UK: Edward Elgar Publishing.

Balconi, M., Fronda, G., Crivelli, D. (2019) Effects of technology-mediated mindfulness practice on stress: psychophysiological and self-report measures, Stress, Vol. 22 No 2, pp. 200-209, DOI: 10.1080/10253890.2018.1531845

Bartlett, L., Martin, A., Neil, A. L., Memish, K., Otahal, P., Kilpatrick, M., \& Sanderson, K. (2019). A systematic review and meta-analysis of workplace mindfulness training randomized controlled trials. Journal of Occupational Health Psychology, Vol. 24 No. 1, pp. 108-126. https://doi.org/10.1037/ocp0000146

Bennike, I. H., Wieghorst, A., and Kirk, U. (2017) Online-based mindfulness training reduces behavioral markers of mind wandering. Journal of Cognitive Enhancement, Vol. 1 No. 2, pp. 172-181. https://doi.org/10.1007/s41465-017-0020-9

Bohlmeijer, E., ten Klooster, P. M., Fledderus, M., Veehof, M., and Baer, R. (2011) Psychometric properties of the five facet mindfulness questionnaire in depressed 
adults and development of a short form. Assessment, Vol. 18 No. 3, pp. 308-320. https://doi.org/10.1177/1073191111408231

Bostock, S., Crosswell, A. D., Prather, A. A., and Steptoe, A. (2019) Mindfulness on-the-go: Effects of a mindfulness meditation app on work stress and well-being. Journal of Occupational Health Psychology, Vol. 24 No. 1, pp. 127-138. https://doi.org/10.1037/ocp0000118

Buck Consultants. (2016) Working well: A global survey of health promotion, workplace wellness, and productivity strategies: A survey report (7th ed., pp. 1-8). Retrieved from https://www.xerox.com/downloads/usa/en/buck/reports/hrc_rp_global_wellbeing_sur vey_2016.pdf

Caesar, A. (2016) Leader gender, relationship quality, and employee perceptions of workplace stress: A correlational study. (Doctor of Philosophy), North Carolina A\&T State University, Greensboro, North Carolina. Available from (UMI No. 10239961) ProQuest Dissertations Publishing database.

Clarke, J., and Draper, S. (2019) Intermittent mindfulness practice can be beneficial, and daily practice can be harmful. An in depth, mixed methods study of the "Calm" app's (mostly positive) effects. Internet Interventions, Vol. 16 No. 19, 100293. https://doi.org/10.1016/j.invent.2019.100293

Cohen, J. (1977). Statistical power analysis for the behavioral sciences. New York: Routledge. 
Cohen, S., and Williamson, G. (1988) Perceived stress in a probability sample of the United States. In S. Spacapan and S. Oskamp (Eds.), The social psychology of health. (pp. 31-68). Newbury Park, CA: Sage.

Creswell, J.D. (2017) Mindfulness Interventions. Annu Rev Psychol. Vol. 3 No. 68, pp. 491516. doi: 10.1146/annurev-psych-042716-051139.

Crivelli, D., Fronda, G., Venturella, I., Balconi, M. (2019) Supporting Mindfulness Practices with Brain-Sensing Devices. Cognitive and Electrophysiological Evidences. Mindfulness, Vol. 10, pp. 301-311. https://doi.org/10.1007/s12671-0180975-3

DeSteno, D., Lim, D., Duong, F., and Condon, P. (2018) Meditation inhibits aggressive responses to provocations. Mindfulness, Vol. 9 No. 4, pp. 1117-1122. https://doi.org/10.1007/s12671-017-0847-2

Eby, L. T., Allen, T. D., Conley, K. M., Williamson, R. L., Henderson, T. G., and Mancini, V. S. (2019) Mindfulness-based training interventions for employees: A qualitative review of the literature. Human Resource Management Review, Vol 29 No. 2, pp. 156-178. https://doi.org/10.1016/j.hrmr.2017.03.004

Economides, M., Martman, J., Bell, M. J., and Sanderson, B. (2018) Improvements in stress, affect, and irritability following brief use of a mindfulness-based smartphone app: A randomized controlled trial. Mindfulness, Vol. 9 No. 5, pp. 1584-1593. https://doi.org/10.1007/s12671-018-0905-4

Fish, J., Brimson, J. \& Lynch, S. (2016) Mindfulness Interventions Delivered by Technology Without Facilitator Involvement: What Research Exists and What Are the Clinical 
Outcomes? Mindfulness, Vol. 7, pp. 1011-1023. https://doi.org/10.1007/s12671-016$0548-2$

Good, D.J., Lyddy, C.J., Glomb, T.M., Bono, J.E., Bown, K.W., Duffy, M.K., Baer, R.A., Brewer, J. A., Lazar, S.W. (2016) Contemplating Mindfulness at Work: An Integrative Review. Journal of Management, Vol. 42 No. 1, pp. 114-142. doi:10.1177/0149206315617003

Gunasekara, A., and Zheng, C. S-M. (2018) Examining the effect of different facets of mindfulness on work engagement. Employee Relations, Vol. 41 No. 1, pp. 193-208. https://doi.org/10.1108/ER-09-2017-0220

Heckenberg, R.A., Eddy, P., Kent, S., Wright, B.J. (2018) Do workplace-based mindfulness meditation programs improve physiological indices of stress? A systematic review and meta-analysis. J Psychosom Res., Vol. 114, pp. 62-71. doi: 10.1016/j.jpsychores.2018.09.010.

Hegney, D., Tsai, L., Craigie, M., Crawford, C., Jay, S., Rees, C., (2020) Experiences of university employees of the impact of a mindful self-care and resiliency program on their well-being, Higher Education Research \& Development. Advance online publication. https://doi.org/10.1080/07294360.2020.1764508

Huberty, J., Green, J., Glissmann, C., Larkey, L., Puzia, M., and Lee, C. (2019) Efficacy of the mindfulness meditation mobile app "calm" to reduce stress among college students: Randomized controlled trial. JMIR Mhealth Uhealth, Vol. 7 No. 6, e14273. https://doi.org/10.2196/14273 
Jamieson, S. D., and Tuckey, M. R. (2017) Mindfulness interventions in the workplace: A critique of the current state of the literature. Journal of Occupational Health Psychology, Vol. 22 No. 2, pp. 180-193. https://doi.org/10.1037/ocp0000048

Kashdan, T. B., Gallagher, M. W., Silvia, P. J., Winterstein, B. P., Breen, W. E., Terhar, D., and Steger, M. F. (2009) The curiosity and exploration inventory-II: Development, factor structure, and psychometrics. Journal of Research in Personality, Vol. 43 No. 6, pp. 987-998. https://doi.org/10.1016/j.jrp.2009.04.011

Kelly, C., (2017) Stress in the higher education sector: causes and yoga-mindfulness interventions. Journal of Yoga and Physiotherapy, Vol. 3 No. 3, pp. 1-14. https://doi.org/10.19080/JYP.2017.03.555613

Kinman, G., \& Johnson, S. (2019). Special section on well-being in academic employees. International Journal of Stress Management, Vol. 26 No. 2, pp. 159161. https://doi.org/10.1037/str0000131

Klatt, M., Buckworth, J., and Malarkey, W. B. (2009) Effects of low-dose mindfulness-based stress reduction (MBSR-ld) on working adults. Health Education and Behavior, Vol. 36 No. 3, pp. 601-614. https://doi.org/10.1177/1090198108317627

Koncz, R., Wolfenden, F., Hassed, C., Chambers, R., Cohen, J., Glozier, N. (2016) Mindfulness-Based Stress Release Program for University Employees. Journal of Occupational and Environmental Medicine, Vol. 58 No. 10, pp. 1021-1027. doi: 10.1097/JOM.0000000000000856

LaMontagne, A.D., Martin, A., Page, K.M., Reavley, K.M., Noblet, A. J., Milner, A.J., Keegal, T., Smith, P.M. (2014) Workplace mental health: developing an integrated 
intervention approach. BMC Psychiatry, Vol. 14 No. 131.

https://doi.org/10.1186/1471-244X-14-131

Lee, K., and Allen, N. J. (2002) Organizational citizenship behavior and workplace deviance: The role of affect and cognitions. Journal of Applied Psychology, Vol. 87 No. 1, pp. 131-142. https://doi.org/10.1037/0021-9010.87.1.131

Leroy, H., Anseel, F., Dimitrova, N. G., and Sels, L. (2013) Mindfulness, authentic functioning, and work engagement: A growth modeling approach. Journal of Vocational Behavior, Vol. 82 No. 3, pp. 238-247. http://doi.org/10.1016/j.jvb.2013.01.012

Lomas, T., Medina, J. C., Ivtzan, I., Rupprecht, S., Hart, R., and Eiroa-Orosa, F. J. (2017) The impact of mindfulness on well-being and performance in the workplace: An inclusive systematic review of the empirical literature. European Journal of Work and Organizational Psychology, Vol. 26 No. 4, pp. 492-513. https://doi.org/10.1080/1359432X.2017.1308924

Malarkey, W. B., Jarjoura, D., and Klatt, M. (2013) Workplace based mindfulness practice and inflammation: A randomized trial. Brain, Behavior, and Immunity, Vol. 27, pp. 145-154. https://doi.org/10.1016/j.bbi.2012.10.009

Michel, A., Bosch, C., and Rexroth, M. (2014) Mindfulness as a cognitive-emotional segmentation strategy: An intervention promoting work-life balance. Journal of Occupational and Organizational Psychology, Vol. 87 No. 4, pp. 733-754. https://doi.org/10.1111/joop.12072

Miksch, D., Lindeman, M. I. H., and Varghese, L. (2015) Minding the mechanisms: A discussion of how mindfulness leads to positive outcomes at work. Industrial and 
Organizational Psychology, Vol. 8 No. 4, pp. 620-629. https://doi.org/10.1017/iop.2015.89

Morrish, E. (2019) Pressure vessels: the epidemic of poor mental health among higher education staff. Occasional paper 20, Higher Education Policy Insitute, Oxford. Available at: https://healthyuniversities.ac.uk/wp-content/uploads/2019/05/HEPIPressure-Vessels-Occasional-Paper-20.pdf

Morrison Wylde, C., Mahrer, N. E., Meyer, R. M. L., and Gold, J. I. (2017) Mindfulness for novice pediatric nurses: Smartphone application versus traditional intervention. Journal of Pediatric Nursing, Vol. 36, pp. 205-212. https://doi.org/10.1016/j.pedn.2017.06.008

Podsakoff, P. M., MacKenzie, S. B., Lee, J.-Y., and Podsakoff, N. P. (2003) Common method biases in behavioral research: A critical review of the literature and recommended remedies. Journal of Applied Psychology, Vol. 88 No. 5, pp. 879-903. https://doi.org/10.1037/0021-9010.88.5.879

Querstret, D., Morison, L., Dickinson, S., Cropley, M., \& John, M. (2020). Mindfulnessbased stress reduction and mindfulness-based cognitive therapy for psychological health and well-being in nonclinical samples: A systematic review and metaanalysis. International Journal of Stress Management. Advance online publication. https://doi.org/10.1037/str0000165

Rich, B. L., Lepine, J. A., and Crawford, E. R. (2010) Job engagement: Antecedents and effects on job performance. Academy of Management Journal, Vol. 53 No. 3, pp. 617635. https://doi.org/10.5465/AMJ.2010.51468988 
Stratton, E., Lampit, A., Choi, I., Calvo, R. A., Harvey, S. B., and Glozier, N. (2017) Effectiveness of eHealth interventions for reducing mental health conditions in employees: A systematic review and meta-analysis. PLoS One, Vol. 12 No. 12, :e0189904. https://doi.org/10.1371/journal.pone.0189904

Valcour, M. (2007) Work-based resources as moderators of the relationship between work hours and satisfaction with work-family balance. Journal of Applied Psychology, Vol. 92 No. 6, pp. 1512-1523. https://doi.org/10.1037/0021-9010.92.6.1512

van Berkel, J., Boot, C. R. L., Proper, K. I., Bongers, P. M., and van der Beek, A. J. (2014) Effectiveness of a worksite mindfulness-related multi-component health promotion intervention on work engagement and mental health: Results of a randomized controlled trial. PLoS One, Vol. 9 No. 1. https://doi.org/10.1371/journal.pone.0084118

van Breukelen, G. J. P. (2006) ANCOVA versus change from baseline had more power in randomized studies and more bias in nonrandomized studies. Journal of Clinical Epidemiology, Vol. 59 No. 9, pp. 920-925. https://doi.org/10.1016/j.jclinepi.2006.02.007

van Emmerik, A. A. P., Berings, F., and Lancee, J. (2018) Efficacy of a mindfulness-based mobile application: A randomized waiting-list controlled trial. Mindfulness, Vol. 9 No. 1, pp. 187-198. https://doi.org/10.1007/s12671-017-0761-7

Virgili, M. (2015) Mindfulness-based interventions reduce psychological distress in working adults: A meta-analysis of intervention studies. Mindfulness, Vol. 6 No. 2, pp. 326337. https://doi.org/10.1007/s12671-013-0264-0 
Vonderlin, R., Biermann, M., Bohus, M., Lyssenko, L. (2020) Mindfulness-based programs in the workplace: a meta-analysis of randomized controlled trials. Mindfulness. Advance online publication. https://doi.org/10.1007/s12671-020-01328-3

Walsh, K. M., Saab, B. J., and Farb, N. A. (2019) Effects of a mindfulness meditation app on subjective well-being: Active randomized controlled trial and experience sampling study. JMIR Mental Health, Vol. 6 No. 1:e10844. https://doi.org/10.2196/10844

Wasil, A. R., Venturo-Conerly, K. E., Shingleton, R. M., and Weisz, J. R., (2019) A review of popular smartphone apps for depression and anxiety: Assessing the inclusion of evidence-based content. Behaviour Research and Therapy, 123. Advance online publication. https://doi.org/10.1016/j.brat.2019.103498

Wayne, S. J., Shore, L. M., and Liden, R. C. (1997) Perceived organizational support and leader-member exchange: A social exchange perspective. Academy of Management Journal, Vol. 40 No. 1, pp. 82-111. https://doi.org/10.2307/257021

West C. P., Dyrbye, L. N., Rabatin, J. T., Call, T. G., Davidson, J. H., Multari, A., Romanski, S. A., Hellyer, J. M., Sloan. J. A., Shanafelt, T, D. (2014) Intervention to promote physician well-being, job satisfaction, and professionalism: A randomized clinical trial. Journal of the American Medical Association Internal Medicine, Vol. 174 No. 4, pp. 527-533. https://doi.org/10.1001/jamainternmed.2013.14387

Wongtongkam, N., Krivokapic-Skoko, B., Duncan, R., Bellio, M. (2017) The influence of a mindfulness-based intervention on job satisfaction and work-related stress and anxiety, International Journal of Mental Health Promotion, Vol. 19 No. 3, pp. 134143. doi: 10.1080/14623730.2017.1316760 
Figure 1: Details of participant flow through the study

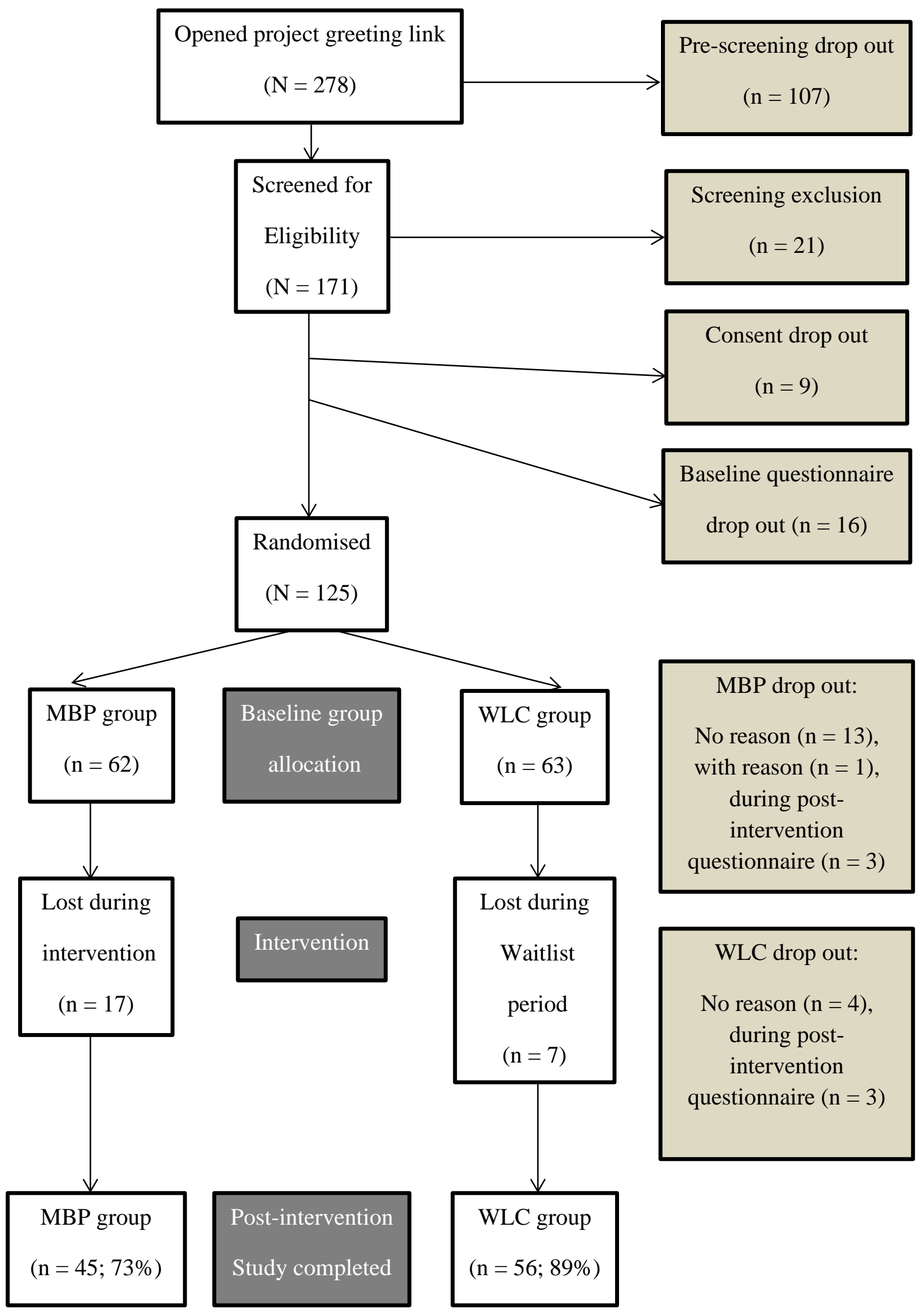


Table 1: Comparison of participant characteristics between groups $(\mathbf{n}=\mathbf{1 2 5})$

\begin{tabular}{|c|c|c|c|c|c|c|}
\hline \multirow[b]{2}{*}{ Characteristic } & \multicolumn{2}{|c|}{ MBP } & \multicolumn{2}{|c|}{ WLC } & \multicolumn{2}{|c|}{ MBP vs WLC } \\
\hline & $n$ & $\%$ & $n$ & $\%$ & $x^{2}(\mathrm{df}=1)$ & $p$ \\
\hline & 62 & & 63 & & & \\
\hline Sex & & & & & 0.700 & .441 \\
\hline Female & 41 & 66.1 & 46 & 73 & & \\
\hline Male & 21 & 33.9 & 17 & 27 & & \\
\hline Age & & & & & 0.197 & .722 \\
\hline$\leq 39$ & 31 & 50 & 34 & 54 & & \\
\hline $40+$ & 31 & 50 & 29 & 46 & & \\
\hline Married or living with a partner & & & & & 0.209 & .691 \\
\hline No & 18 & 29 & 16 & 25.4 & & \\
\hline Yes & 44 & 71 & 47 & 74.6 & & \\
\hline Education level & & & & & 1.285 & .283 \\
\hline No university degree & 16 & 25.8 & 11 & 17.5 & & \\
\hline University degree & 46 & 74.2 & 52 & 82.5 & & \\
\hline Stress levels & & & & & 1.264 & .541 \\
\hline Low & 6 & 9.7 & 10 & 15.9 & & \\
\hline Moderate & 34 & 54.8 & 30 & 47.6 & & \\
\hline High & 22 & 35.5 & 23 & 36.5 & & \\
\hline Occupation & & & & & 0.104 & .848 \\
\hline Academic and research & 19 & 30.6 & 21 & 33.3 & & \\
\hline Back-office and support & 43 & 69.4 & 42 & 66.7 & & \\
\hline Work location & & & & & 0.453 & .540 \\
\hline On-site & 45 & 72.6 & 49 & 77.8 & & \\
\hline Some remote work & 17 & 27.4 & 14 & 22.2 & & \\
\hline Any mindfulness/meditation pra & year & & & & 0.082 & .808 \\
\hline No & 52 & 83.9 & 54 & 85.7 & & \\
\hline Yes & 10 & 16.1 & 9 & 14.3 & & \\
\hline
\end{tabular}

$\mathrm{MBP}=$ mindfulness program group; $\mathrm{WLC}=$ waitlist control group; Stress level categories are based on the participant Perceived Stress Scores at baseline and categorized by the average level for their age (Caesar, 2016) 
Table 2: Baseline outcome variable comparisons between groups $(\mathbf{n = 1 2 5})$

\begin{tabular}{|c|c|c|c|c|}
\hline \multirow[b]{2}{*}{ Study variables } & \multicolumn{2}{|c|}{ Group means } & \multicolumn{2}{|c|}{$t$-test } \\
\hline & $\begin{array}{c}\text { MBP } \\
\text { Mean (SD) }\end{array}$ & $\begin{array}{c}\text { WLC } \\
\text { Mean (SD) }\end{array}$ & $t(123)$ & $p$ \\
\hline & $\mathrm{n}=62$ & $\mathrm{n}=63$ & & \\
\hline \multicolumn{5}{|l|}{ Mindfulness } \\
\hline Observe & $12.87(3.84)$ & $11.83(3.42)$ & 1.607 & .111 \\
\hline Describing & $14.90(2.08)$ & $15.44(1.99)$ & -1.487 & .140 \\
\hline Acting with awareness & $15.73(4.29)$ & $15.29(4.09)$ & 0.587 & .558 \\
\hline Nonjudgment & $15.21(4.96)$ & $15.89(4.68)$ & -.788 & .432 \\
\hline Nonreactivity & $13.69(4.11)$ & $13.19(3.69)$ & 0.721 & .472 \\
\hline Total Mindfulness & $72.40(12.04)$ & $71.63(11.50)$ & 0.365 & .716 \\
\hline \multicolumn{5}{|l|}{ Health related outcome } \\
\hline Perceived stress & $22.00(6.85)$ & $21.21(7.20)$ & 0.632 & .528 \\
\hline \multicolumn{5}{|l|}{ Work related outcomes } \\
\hline Work-life balance (WLB) & $15.76(4.44)$ & $15.79(4.57)$ & -.044 & .965 \\
\hline Job Engagement: Physical & $24.24(4.27)$ & $24.6(3.92)$ & -0.493 & .623 \\
\hline Job Engagement: Emotional & $21.71(4.92)$ & $23.24(4.34)$ & -1.843 & .068 \\
\hline Job Engagement: Cognitive & $23.71(4.21)$ & $23.87(4.16)$ & 0.218 & .828 \\
\hline Total Job Engagement & $69.66(11.73)$ & $71.71(10.89)$ & -1.014 & .313 \\
\hline Organizational behavior & $40.08(7.74)$ & $39.46(7.20)$ & 0.464 & .643 \\
\hline Curiosity: Explore & $17.15(4.33)$ & $17.94(3.84)$ & -1.083 & .281 \\
\hline Curiosity: Embrace & $13.40(4.31)$ & $14.21(4.06)$ & -1.073 & .286 \\
\hline Total Curiosity & $30.55(8.15)$ & $32.14(7.51)$ & -1.138 & .257 \\
\hline Intention to quit & $14.81(7.47)$ & $13.41(7.44)$ & 1.045 & .298 \\
\hline
\end{tabular}

$\mathrm{MBP}=$ mindfulness program group; $\mathrm{WLC}=$ waitlist control group 
Table 3: Summary of Headspace usage data for the MBP group

\begin{tabular}{lcccccc}
\hline & \multicolumn{2}{c}{ ITT } & \multicolumn{2}{c}{ Completers } & \multicolumn{2}{c}{ Practitioners } \\
& $\mathbf{n}$ & $\boldsymbol{\%}$ & $\mathbf{n}$ & $\boldsymbol{\%}$ & $\mathbf{n}$ & $\boldsymbol{\%}$ \\
\hline Total participants & 62 & 100 & 45 & 100 & 31 & 100 \\
Number of Headspace foundation sessions & & & & & \\
0 & 17 & 27 & 5 & 11 & - & - \\
$1-5$ & 6 & 10 & 3 & 7 & - & - \\
$6-10$ & 35 & 56 & 33 & 73 & 27 & 87 \\
$11-20$ & 4 & 7 & 4 & 9 & 4 & 13 \\
& & & & & & \\
Total number of Headspace sessions & & & & & & \\
0 & 17 & 27 & 5 & 11 & - & - \\
$1-5$ & 4 & 7 & 2 & 4 & - & - \\
$6-10$ & 12 & 19 & 10 & 22 & 3 & 10 \\
$11-20$ & 8 & 13 & 7 & 16 & 7 & 23 \\
$21-40$ & 11 & 18 & 11 & 24 & 11 & 35 \\
$41-60$ & 5 & 8 & 5 & 11 & 5 & 16 \\
$>60^{\text {a }}$ & 5 & 8 & 5 & 11 & 5 & 16 \\
Total duration of Headspace practice & & & & & & \\
$0-10$ minutes & 17 & 27 & 5 & 11 & - & - \\
11 mins -1 hour & 8 & 13 & 5 & 11 & - & - \\
$1.01-2$ hours & 9 & 14 & 7 & 16 & 3 & 10 \\
$2.01-3$ hours & 6 & 10 & 6 & 13 & 6 & 19 \\
$3.01-5$ hours & 5 & 8 & 5 & 11 & 5 & 16 \\
$5.01-8$ hours & 7 & 11 & 7 & 16 & 7 & 23 \\
$8.01-11$ hours & 7 & 11 & 7 & 16 & 7 & 23 \\
$>11$ hours & 3 & 5 & 3 & 7 & 3 & 10 \\
\hline
\end{tabular}

${ }^{a}$ The maximum number of sessions was 85

${ }^{\mathrm{b}}$ The maximum total duration of practice was 14 hours and 40 minutes 
Table 4: Post program outcome variable comparisons between groups - ITT analysis $(\mathbf{n = 1 2 5 )}$

\begin{tabular}{|c|c|c|c|c|c|c|c|c|}
\hline \multirow[b]{3}{*}{ Study variables } & \multicolumn{2}{|c|}{ Group means } & \multicolumn{6}{|c|}{ ANCOVA } \\
\hline & \multirow{2}{*}{$\begin{array}{c}\text { MBP } \\
\text { Mean (SD) }\end{array}$} & \multirow{2}{*}{$\begin{array}{c}\text { WLC } \\
\text { Mean (SD) }\end{array}$} & \multirow[b]{2}{*}{ difference } & \multicolumn{2}{|c|}{$95 \% \mathrm{CI}$} & \multirow[b]{2}{*}{$F(1,122)$} & \multirow[b]{2}{*}{$p$} & \multirow[b]{2}{*}{ Cohen's $d$} \\
\hline & & & & lower & upper & & & \\
\hline \multicolumn{9}{|l|}{ Mindfulness } \\
\hline Observe & $13.89(3.85)$ & $12.46(3.77)$ & 0.60 & -0.30 & 1.50 & 1.75 & .188 & 0.24 \\
\hline Nonjudgment & $15.85(4.77)$ & $15.54(4.84)$ & 0.88 & -0.08 & 1.83 & 3.32 & .071 & 0.33 \\
\hline Nonreactivity & $14.73(3.94)$ & $13.29(4.32)$ & 1.03 & 0.10 & 1.96 & 4.78 & .031 & 0.39 \\
\hline Total Mindfulness & $76.69(12.48)$ & $72.16(12.59)$ & 3.89 & 1.13 & 6.66 & 7.76 & .006 & 0.50 \\
\hline \multicolumn{9}{|l|}{ Health related outcome } \\
\hline Perceived stress & $19.13(6.68)$ & $20.44(7.33)$ & -1.89 & -3.63 & -0.15 & 4.60 & .034 & -0.39 \\
\hline \multicolumn{9}{|l|}{ Work related outcomes } \\
\hline Job Engage: Physical & $24.27(4.21)$ & $24.33(4.45)$ & 0.24 & -0.74 & 1.22 & 1.23 & .634 & 0.09 \\
\hline Job Engage: Emotional & $22.18(4.86)$ & $22.84(4.99)$ & 0.62 & -0.47 & 1.71 & 1.26 & .263 & 0.20 \\
\hline Job Engage: Cognitive & $23.71(4.15)$ & $24.19(4.25)$ & -0.36 & -1.42 & 0.69 & 0.47 & .494 & -0.12 \\
\hline Total Job Engagement & $70.16(11.53)$ & 71.37 (12.14) & 0.49 & -2.11 & 3.09 & 0.14 & .709 & 0.07 \\
\hline Organizational behavior & $40.53(7.19)$ & $39.90(8.22)$ & 0.13 & -1.59 & 1.84 & 0.02 & .885 & 0.03 \\
\hline Curiosity - Explore & $17.10(4.19)$ & $17.60(3.77)$ & 0.15 & -0.62 & 0.91 & 0.01 & .709 & 0.07 \\
\hline Curiosity - Embrace & $13.94(4.36)$ & $14.25(4.38)$ & 0.38 & -0.48 & 1.24 & 0.76 & .384 & 0.16 \\
\hline Total Curiosity & 31.03 (8.17) & $31.86(7.75)$ & 0.56 & -0.91 & 2.04 & 0.57 & .452 & 0.14 \\
\hline Intention to quit & $14.95(7.94)$ & $15.35(8.87)$ & -1.63 & -3.50 & 0.24 & 2.97 & .087 & -0.31 \\
\hline
\end{tabular}

$\mathrm{MBP}=$ mindfulness program group; WLC $=$ waitlist control group 
Table 5: Post program outcome variable comparisons between groups - supplementary analysis: completers and practitioners

\begin{tabular}{|c|c|c|c|c|c|c|c|c|c|c|c|c|}
\hline \multirow[b]{3}{*}{ Study variables } & \multicolumn{6}{|c|}{ ANCOVA (completers $n=101$ ) } & \multicolumn{6}{|c|}{ ANCOVA (practitioners $\mathrm{n}=87$ ) } \\
\hline & \multicolumn{4}{|c|}{$95 \%$ CI } & \multirow[b]{2}{*}{$P$} & \multirow[b]{2}{*}{ Cohen's $d$} & \multicolumn{3}{|c|}{$95 \% \mathrm{CI}$} & \multirow[b]{2}{*}{$F(1,84)$} & \multirow[b]{2}{*}{$P$} & \multirow[b]{2}{*}{ Cohen's $d$} \\
\hline & difference & lower & upper & $F(1,98)$ & & & difference & lower & upper & & & \\
\hline \multicolumn{13}{|l|}{ Mindfulness } \\
\hline Observe & 0.99 & -0.11 & 2.09 & 3.21 & .076 & 0.37 & 1.02 & -0.18 & 2.22 & 2.85 & .095 & 0.38 \\
\hline Describing & 0.13 & -0.39 & 0.64 & 0.24 & .624 & 0.10 & 0.27 & -0.30 & 0.84 & 0.88 & .351 & 0.21 \\
\hline Acting with awareness & 1.67 & 0.50 & 2.83 & 8.04 & .006 & 0.58 & 1.74 & 0.39 & 3.09 & 6.58 & .012 & 0.57 \\
\hline Nonjudgment & 1.35 & 0.21 & 2.50 & 5.47 & .021 & 0.47 & 1.99 & 0.72 & 3.25 & 9.75 & .002 & 0.70 \\
\hline Nonreactivity & 1.42 & 0.29 & 2.54 & 6.25 & .014 & 0.51 & 1.42 & 0.13 & 2.71 & 4.80 & .031 & 0.50 \\
\hline Total Mindfulness & 5.55 & 2.22 & 8.87 & 10.96 & .001 & 0.67 & 6.52 & 2.74 & 10.29 & 11.77 & .001 & 0.77 \\
\hline \multicolumn{13}{|l|}{ Health related outcome } \\
\hline Perceived stress & -2.63 & -4.67 & -0.59 & 6.54 & .012 & -0.52 & -3.15 & -5.44 & -0.86 & 7.47 & .008 & -0.55 \\
\hline \multicolumn{13}{|l|}{ Work related outcomes } \\
\hline Work-life balance (WLB) & 1.11 & -0.33 & 2.56 & 2.35 & .129 & 0.31 & 1.69 & 0.05 & 3.33 & 4.17 & .044 & 0.46 \\
\hline Job Engage: Physical & 0.51 & -0.69 & 1.71 & 0.72 & .400 & 0.17 & 0.51 & -0.84 & 1.86 & 0.57 & .454 & 0.17 \\
\hline Job Engage: Emotional & 0.76 & -0.55 & 2.06 & 1.32 & .253 & 0.23 & 1.48 & 0.03 & 2.94 & 4.13 & .045 & 0.46 \\
\hline Job Engage: Cognitive & 0.11 & -1.17 & 1.38 & 0.03 & .871 & 0.03 & -0.01 & -1.45 & 1.42 & 0.01 & .986 & 0.00 \\
\hline Total Job Engagement & 1.12 & -2.02 & 4.26 & 0.50 & .481 & 0.14 & 1.76 & -1.79 & 5.32 & 0.97 & .327 & 0.22 \\
\hline Organizational behavior & 0.16 & -1.91 & 2.24 & 0.02 & .878 & 0.03 & 0.53 & -1.73 & 2.80 & 0.22 & .640 & 0.11 \\
\hline Curiosity: Explore & 0.20 & -0.71 & 1.12 & 0.20 & .659 & 0.09 & 0.50 & -0.55 & 1.54 & 0.89 & .347 & 0.21 \\
\hline Curiosity: Embrace & 0.55 & -0.50 & 1.60 & 1.07 & .303 & 0.21 & 0.96 & -0.22 & 2.15 & 2.61 & .110 & 0.35 \\
\hline Total Curiosity & 0.75 & -1.03 & 2.53 & 0.71 & .402 & 0.17 & 1.45 & -0.57 & 3.47 & 2.04 & .157 & 0.32 \\
\hline Intention to quit & -1.73 & -4.05 & 0.60 & 2.17 & .144 & -0.30 & -2.31 & -4.88 & 0.26 & 3.18 & .078 & -0.40 \\
\hline
\end{tabular}

$\mathrm{MBP}=$ mindfulness program group; WLC $=$ waitlist control group 